\title{
Autophagy Facilitates Metadherin-Induced Chemotherapy Resistance Through the AMPK/ATG5 Pathway in Gastric Cancer
}

\author{
Guoqing Peia Meng Luo ${ }^{a} \quad$ Xiaochun Nia Jugang Wu ${ }^{a}$ Shoulian Wang ${ }^{a}$ \\ Yiwen $\mathrm{Ma}^{\mathrm{b}}$ Jiwei $\mathrm{Yu}^{\mathrm{a}}$ \\ aDepartment of General Surgery, Shanghai Ninth People's Hospital, School of Medicine, Shanghai Jiao \\ Tong University, Shanghai, 'Department of Anaesthesiology, Shanghai Ninth People's Hospital, School \\ of Medicine, Shanghai Jiao Tong University, Shanghai, China
}

\section{Key Words}

Mtdh • Chemoresistance $\cdot$ Autophagy $•$ Gastric cancer

\begin{abstract}
Background/Aims: Metadherin (MTDH) is overexpressed in some malignancies and enhances drug resistance; however, its role in gastric cancer $(\mathrm{GC})$ and the underlying mechanisms remain largely unexplored. Here, we explore the mechanism by which MTDH induces drug resistance in GC. Methods: We analysed the level of MTDH in GC and adjacent normal gastric mucosal tissues by real-time quantitative PCR (q-PCR). We also analysed the level of autophagy by western blot analysis, confocal microscopy, and transmission electron microscopy after MTDH knockdown and overexpression, and examined fluorouracil (5-FU) resistance by Cell Counting Kit-8 at the same time. Finally, GC patient-derived xenograft tumours were used to demonstrate 5-FU resistance. An AMPK pathway inhibitor was applied to determine the molecular mechanisms of autophagy. Results: MTDH expression was significantly increased in the GC specimens compared with that in the adjacent normal gastric mucosal tissues. Further study showed a positive correlation between the expression level of MTDH and 5-FU resistance. MTDH overexpression in MKN45 cells increased the levels of P-glycoprotein (Pgp) and promoted 5-FU resistance, while inhibition of MTDH showed the opposite result. The simultaneous inhibition of autophagy and overexpression of MTDH decreased the levels of P-gp and inhibited 5-FU resistance. Moreover, MTDH induced AMPK phosphorylation, regulated ATG5 expression, and finally influenced autophagy, suggesting that MTDH may activate autophagy via the AMPK/ATG5 signalling pathway. Our findings reveal a unique mechanism by which MTDH promotes GC chemoresistance and show that MTDH is a potential target for improved chemotherapeutic sensitivity and GC patient survival. Conclusions: MTDH-stimulated cancer resistance to 5-FU may be mediated through autophagy activated by the AMPK/ATG5 pathway in GC.
\end{abstract}

G. Pei and M. Luo contributed equally to this work.

Jiwei Yu

and Yiwen Ma 


\section{Cellular Physiology Cell Physiol Biochem 2018;46:847-859

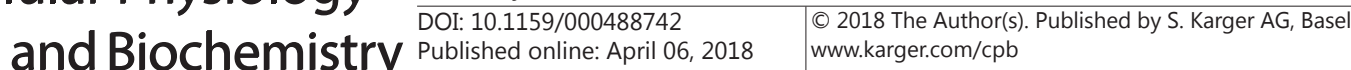

Pei et al.: Autophagy Facilitates Chemotherapy Resistance in GC

\section{Introduction}

Gastric cancer (GC) is the fourth most frequently occurring malignancy and remains the second leading cause of cancer death worldwide [1]. Surgery is the foremost treatment. Although the survival rates of GC patients have improvedin combination with chemoradiotherapy, the outcomes remain poor [2]. Chemotherapy is the most effective treatment after surgery and significantly improves the survival rates of GC patients. Nevertheless, drug resistance remains a majorobstacle for GC treatment. Therefore, exploration of the mechanisms underlying chemoresistance may contribute to more effective handling of GC patients and thus improve their survival.

Astrocyte elevated gene-1 (AEG-1), which is called metadherin (MTDH) in humans [3] and lysine-rich CEACAM-1-associated protein (LYRIC) in rats [4], is a single transmembrane protein. MTDH is located at chromosome 8q22.5, is composed of 582 amino acids [5], and has been identified as an oncogene. MTDH is induced in primary human foetal astrocytes by HIV-1 and tumour necrosis factor- $\alpha$ and has been reported to have elevated expression in more than 20 types of cancer [6], including hepatocellular carcinoma, GC, and breast cancer [7-13]. Our previous study reported that MTDH promoted tumourigenesis in the late phase of GC development partially through the induction of epithelial-to-mesenchymaltransition [14], which contributed to GC progression, invasion, and metastasis. MTDH also participates actively in autophagy and chemoresistance [15-18].

Autophagy is a protective mechanism in normal cells and has the same protective effect when a tumour develops. Autophagy is an evolutionarily conserved process by which longlived and functionally redundant proteins are degraded in the cell or intracellular organelles are decomposed to provide cellular energy and the building blocks for metabolism [19]. Autophagy occurs when cells are confronted with nutrient starvation, xenobiotic treatment, and other environmental stressors [20].

Recently, MTDH has been suggested to have potentially crucial and broad functionality in human cancer [21]. MTDH has been reported to activate the expression of the multidrug resistance gene 1 (MDR1) protein [22]. Autophagy has also been shown to enhance drug resistance [23]; however, whether MTDH can mediate resistance to the anti-cancer drug fluorouracil (5-FU) through autophagy has not been investigated [24].

Autophagy-related genes (Atgs) are a group of proteins that play crucial roles in autophagy. Atg5 is important for cell development and is involved in autophagosome formation in the early stages by conjugating Atg12 with Atg8 (LC3) [25]. A variety of cellular signalling pathways are regulated by MTDH, including PI3K/AKT nuclear factor-kappa B, Wnt/ $\beta$-catenin, and MAPK/ERK [26]. Cheng et al. found that MTDH mediated trastuzumab resistance through an NF- $\kappa B$-dependent pathway and decreased PTEN expression [24].Sujit et al. noted that MTDH enhanced autophagy via a non-classical pathway. MTDH promotes AMPK phosphorylation, increases ATG5 expression, and consequently facilitates autophagy. However, the molecular mechanisms by which MTDH promotes autophagy and drug resistance in GC have not been fully investigated [27].

MTDH plays a key role in the development of drug resistance. In this study, we address whether MTDH plays an important role in the development of resistance to chemotherapy in GC and evaluate whether autophagy is involved in this process. We found that MTDH enhanced the drug resistance of gastric MKN45 cells via autophagy through the AMPK/ATG5 pathway. Moreover, our findings provide insights into how MTDH controls ATG5 expression and activity during the development of chemotherapy resistance in GC and its underlying mechanism. This study lays the foundation for a therapeutic target by inhibiting MTDH in clinical GC patients and promotes the development of effective treatment strategies. 


\section{Cellular Physiology Cell Physiol Biochem 2018;46:847-859 \begin{tabular}{l|l} 
and Biochemistry Published ondis: Apri 06, 2018 & $\begin{array}{l}\text { C } 2018 \text { The Author(s). Published by S. Karger AG, Basel } \\
\text { www.karger.com/cpb }\end{array}$
\end{tabular}}

Pei et al.: Autophagy Facilitates Chemotherapy Resistance in GC

\section{Materials and Methods}

\section{Clinical tissue collection}

Under approval from the Shanghai Ninth People's Hospital Ethics Committee, 92 GC tissues and their corresponding para-cancerous tissues ( $2 \mathrm{~cm}$ away from the cancer) were selected after each patient signed an informed consent form. All patients were treated by GC radical correction at Shanghai Ninth People's Hospital from 2015 to 2016 and did not accept chemoradiotherapy prior to surgery. The tissues were segmented into 3 parts and stored at $-80^{\circ} \mathrm{C}$ prior to measuring mRNA expression levels; the segments were fixed in $10 \%$ neutral-buffered formalin for immunohistochemical staining and were frozen in liquid nitrogen for the preparation of GC patient-derived xenograft (PDX) animal models.

\section{Cell lines and cell culture}

The human GC AGS, SGC7901, BGC823, HGC803, and MKN45 cell lines and the human normal gastric mucosa GES-1 cell line were obtained from the American Type Culture Collection (Manassas, VA, USA). According to the manufacturer's instructions, the cells were cultured in 90\% RPMI medium (Gibco, Grand Island, NY, USA) supplemented with 10\% foetal bovine serum (Gibco), except for the GES-1 cell line, which was cultured in 90\% Dulbecco's modified Eagle's medium (Gibco) containing 10\% foetal bovine serum. All cell lines were cultivated in a humidified atmosphere at $37^{\circ} \mathrm{C}$ with $5 \% \mathrm{CO}_{2}$.

\section{siRNA interference and stable knockdown cell line establishment}

The MTDH knockdown sequence was 5'-GCCAUCUGUAAUCUUAUCATT-3', the ATG5 knockdown sequence was 5'-GTCCATCTAAGGATGCAAT-3', and the AMPK knockdown sequence was 5'-GCGUGUACGAAGGAAGAAUTT-3'. A scrambled targeting sequence of these gene sequences was used as a negative control, separately. Stable knockdown and overexpression MKN45 cell lines were established according to our previous study [14].

\section{Cell viability assay}

The MKN45-sh and MKN45-MTDH and their control cell lines were seeded into 96-well plates at a density of $1.0 \times 10^{4}$ cells per well. The exterior wells surrounding the plates were filled with phosphatebuffered saline (as the control) to reduce error. At $24 \mathrm{~h}$ later, the medium was replaced with $200 \mu \mathrm{L}$ fresh medium, and the cells were treated with different concentrations of 5-FU $(0,1,10,100$, and $1000 \mu \mathrm{mol} / \mathrm{L})$ for $48 \mathrm{~h}$. Then, the medium was discarded, and $100 \mu \mathrm{L}$ fresh medium was mixed with $10 \mu \mathrm{L}$ Cell Counting Kit-8 (CCK-8; Dojindo, Kumamoto, Japan) solution and added to each well. After $2 \mathrm{~h}$ of cultivation in a $37^{\circ} \mathrm{C}$ incubator, the absorbance at $450 \mathrm{~nm}$ was measured on a Tecan Infinite M200 PRO (Grödig, Austria). The impact of background absorbance was eliminated by using the absorbance percentage of drug-treated cells relative to untreated cells to obtain cell viability. The 50\% inhibitory concentration (IC50) values for the cells under different handling conditions were determined using nonlinear regression analysis with SPSS 20.0 software.

\section{Transmission electron microscopy}

MKN45 cells were infected with the AEG-1 expression plasmid for $48 \mathrm{~h}$. The MKN45-sh and MKN45MTDH cell lines and their blank controls were immobilized with glutaraldehyde for $12 \mathrm{~h}$ at $4^{\circ} \mathrm{C}$. The cells were embedded, sectioned, and then analysed by transmission electron microscopy. The structure of the embedded organelles in a double membrane package (autophagolysosome) was observed and photographed.

\section{Confocal microscopy}

All cell types were seeded into 6-well plates including a cell climbing piece at a density of $2.0 \times 10^{5}$ cells per well. After $24 \mathrm{~h}$, the RFP-GFP-LC3 plasmid ( $4 \mu \mathrm{g}$ per well) was transfected into the above cells via liposomes using the Invitrogen Lipofectamine 3000 reagent. At $48 \mathrm{~h}$ later, the cells were immobilised using $4 \%$ paraformaldehyde for $10 \mathrm{~min}$, washed 3 times with phosphate-buffered saline, andfluoromounted with Fluoromount-G containing 4',6-diamidino-2-phenylindole for $5 \mathrm{~min}$ at $37^{\circ} \mathrm{C}$ in the dark. The samples were observed under a ZEISS LSM710 microscope with ZEN 2009 Light Edition software. 


\section{Cellular Physiology Cell Physiol Biochem 2018;46:847-859 \begin{tabular}{l|l} 
and Biochemistry Publis.1159/000488742 & $\begin{array}{l}\text { (c) 2018 The Author(s). Published by S. Karger AG, Basel } \\
\text { www.karger.com/cpb }\end{array}$
\end{tabular} \\ Pei et al.: Autophagy Facilitates Chemotherapy Resistance in GC}

\section{Histology and immunohistochemistry}

MTDH expression in tumour tissues was evaluated using immunohistochemistry. Serial 4- $\mu$ m-thick tissue sections were cut, deparaffinised, rehydrated, and then heat-treated for $30 \mathrm{~min}$ in citrate buffer $\mathrm{pH}$ 6.0) for antigen retrieval. Next, $3 \% \mathrm{H}_{2} \mathrm{O}_{2}$ was used to block endogenous peroxidase activity for $10 \mathrm{~min}$. The sections were incubated with an anti-MTDH monoclonal antibody (1:5000 dilution; Abcam, Cambridge, UK) overnight after blocking with 5\% normal blocking serum. The next day, the sections were incubated with a biotinylated secondary antibody (Boster, Wuhan, China) for $40 \mathrm{~min}$, followed by anstreptavidin-biotin complex solution according to the manufacturer's instructions. The sections were subsequently observed under a microscope. The intensity of protein expression was assessed using the $\mathrm{H}$-score as follows: $\mathrm{H}$-score $=\sum(\mathrm{Pi} \times \mathrm{I}) / 100$, where Pi denotes the percentage of stained cells, and I denotes the staining intensity with a range from 1 to 3 . The H-score ranged from 1 to 300 .

\section{$q-P C R$}

Total RNA was extracted from GC cells with the TRIzol reagent (Invitrogen, Carlsbad, CA, USA) under the guidance of the manufacturer's protocol. The concentration of the isolated RNA was measured with a NanoDrop 2000 spectrophotometer (Thermo Fisher Scientific, Waltham, MA, USA) and then reverse transcribed to obtain complementary DNA using a RevertAid ${ }^{\mathrm{TM}}$ First Strand cDNA Synthesis Kit (Takara Bio, Shiga, Japan). q-PCR was performed using SYBR Green I Master Mix (Biotool, Houston, TX, USA) in a LightCycler 480 (Roche, Eugene, OR, USA) and analysed with LightCycler 480 Software (release 1.5.1.62 SP2). The following primer pairs were used: AEG-1: 5'-AAGCAGTGCAAAACAGTTCACG-3' (forward) and 5'-GCACCTTATCACGTTTACGCT-3' (reverse); ATG5: 5'-GGCCATCAATCGGAAACTC-3' (forward) and 5'-CAGCCACAGGACGAAACAG-3' (reverse); PRKAA1: 5'-GACAGCCGAGAAGCAGAAAC-3' (forward) and 5'-CCAGTCAATTCATGTTTGCCA-3' (reverse); MDR1: 5'-ACTCACTTCAGGAAGCAACCAG-3' (forward) and 5'-TGATTCCTCGAGAAACTGCG-3' (reverse); and GAPDH (internal control): 5'-AAGGTGAAGGTCGGAGTCAA-3' (forward) and $5^{\prime}$-GGAAGATGGTGATGGGATTT-3' (reverse). Relative mRNA expression levels were determined using the comparative Ct method after normalising to GAPDH expression levels.

\section{Western blotting analysis}

The following antibodies were used for the western blotting analysis: LYRIC (1:5000 dilution; Abcam, ab124789), AMPK (1:1000 dilution; Cell Signaling Technology, Danvers, MA, USA, 5831T), P-AMPK (1:1000 dilution; Cell Signaling Technology, 2535T), ATG5 (1:1000 dilution; Cell Signaling Technology, 2630S), LC3 (1:1000 dilution; Cell Signaling Technology, 2775S), GAPDH (1:5000 dilution; Bioworld Technology, Minneapolis, MN, USA), and P-glycoprotein (P-gp, 1:500 dilution; Abcam, ab170904).

The cells were lysed with radioimmunoprecipitation assaylysis buffer mixed with aphenylmethanesulfonyl fluoride protease inhibitor at a 100:1 ratio (Beyotime Institute of Biotechnology, Shanghai, China). The protein concentrations were determined using a Bicinchoninic Acid Protein Assay Kit (Beyotime Institute of Biotechnology).

Equal amounts of protein were separated by sodium dodecyl sulphate-polyacrylamide gel electrophoresis and then transferred to polyvinylidenedifluoride membranes $(0.22-\mu \mathrm{m}$; Millipore, Billerica, MA, USA). The membranes were blocked in 5\% non-fat milk with TBST for $2 \mathrm{~h}$ at room temperature and then incubated with the appropriate primary antibodies at $4^{\circ} \mathrm{C}$ overnight. After washing 3 times with TBST for $10 \mathrm{~min}$ per wash, the membranes were incubated with horseradish peroxidase-conjugated secondary antibodies (1:5000; Bioworld Technology, St. Louis Park, MN, USA) at room temperature for $1 \mathrm{~h}$, except for GAPDH, which was already conjugated with the primary antibody. Finally, the protein bands were detected with an Enhanced Chemiluminescence Detection Kit (Thermo Scientific, Waltham, MA, USA) and photographed with the Gel Logic 2200 PRO imaging system.

\section{Experimental animals (PDX tumour models)}

Sixty-five-week-old male BALB/c nude mice were used to construct the GC PDX tumour models. GC tissues with high and low MTDH expression levels (5 tissues each) were inoculated subcutaneously into the abdomens of the nude mice, with a piece of dimensions $2 \mathrm{~mm} \times 2 \mathrm{~mm} \times 2 \mathrm{~mm}$ used to prepare the inoculations for 6 mice. When lump growth reached $5 \mathrm{~mm} \times 5 \mathrm{~mm}, 3$ mice for every tissue source (ie, case) were considered the control group and received a saline injection into the abdominal cavityonce every 2 days. The other 3 mice were injected with $5-\mathrm{FU}(5 \mathrm{mg} / \mathrm{kg})$. The short and long diameters of the tumours 
Pei et al.: Autophagy Facilitates Chemotherapy Resistance in GC

were measured every other day. Tumour volume $=(\text { short diameter })^{2} \times($ long diameter $) / 2$ [28]. After 12 days of injection, the mice were euthanized, and the tumours were fixed in $4 \%$ formaldehyde for additional examinations. All animal procedures were performed in agreement with the institutional guidelines.

The mice were housed under specific pathogen-free conditions with a 12-h light and 12-h dark cycle, and food and water were provided routinely. All experiments were performed with approval from the Animal Care and Use Committee of Shanghai Ninth Hospital, affiliated with the Medical College of Shanghai Jiao Tong University (Shanghai, China). All efforts were made to minimise the number of experimental animals and their suffering.

\section{Statistical analysis}

Statistical analysis was performed using SPSS 19.0 software (IBM Corporation, New York, NY, USA) or GraphPad Prism software 6.0 (GraphPad Software, Inc., San Diego, CA, USA). The data are presented as the mean \pm standard deviation (SD)and $\mathrm{P}<0.05$ was considered significant $\left({ }^{*} \mathrm{P}<0.05\right)$. All operations were performed in accordance with the Student's t test or one-way analysis of variance.

\section{Results}

Increased MTDH expression is correlated with GC patients

MTDH expression levels were compared between GC and adjacent normal gastric mucosal tissues obtained from $92 \mathrm{GC}$ patients by immunohistochemical staining. Compared with the adjacent normal gastric mucosal tissues, $84 \mathrm{GC}$ tissues were found to have increased MTDH expression, and 8 GC tissues had decreased MTDH expression based on immunohistochemical staining (Fig. 1A). The H-scores were calculated for the GC and para-cancerous tissues according to the immunohistochemical staining results; the $\mathrm{H}$-scores were significantly higher for MTDH in the cancer tissues than those in the para-cancerous tissues (Fig. $1 \mathrm{~B}$ and $1 \mathrm{C}$ ). In our previous study, MTDH expression levels were related to lymph node metastasis; tumour, node, metastasis stage; and median overall survival time [14]. Thus, increased MTDH expression is correlated with GC patient survival.

We randomly selected 40 pairs of GC and para-cancerous tissues. Compared with the MTDH mRNA expression levels in para-cancerous tissues, those in 36 GC tissues were increased and those in 4 GC tissues were decreased; ATG5 mRNA expression was increased in 38 GC tissues and decreased in 2 GC tissues (Fig. 1D and 1E).

We divided the abovementioned $92 \mathrm{GC}$ tissue samples into 2 groups based on low and high MTDH expression. Five tissue samples from each group were selected to establish PDX tumour models (see section 3.6).

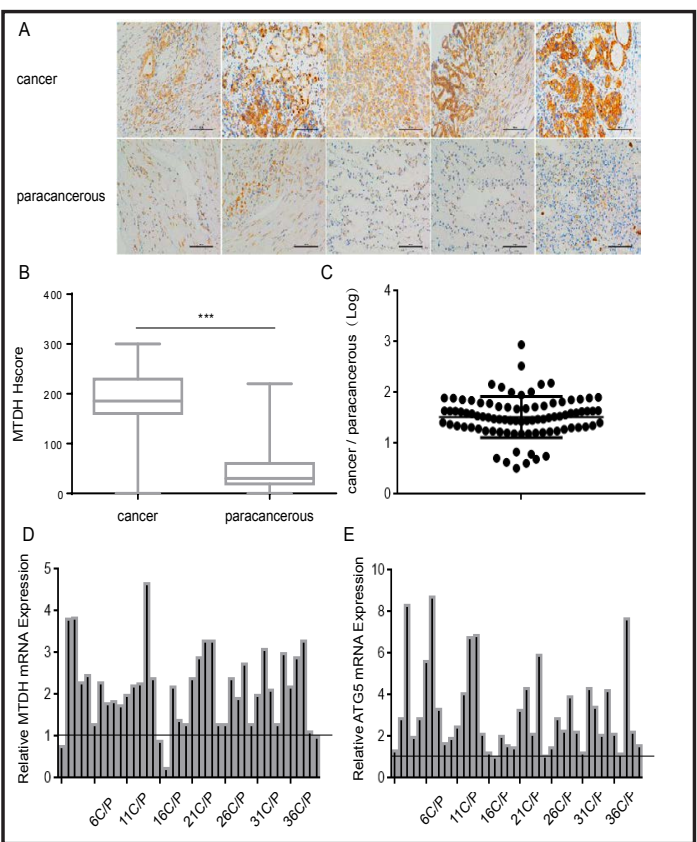

Fig. 1.MTDH expression in GC and para-cancerous gastric mucosal tissues. (A) Immunohistochemistry staining for MTDH expression in para-cancerous gastric mucosal and primary GC tissues. Scale bar, $100 \mu \mathrm{m}$. (B and C) MTDH H-scores for 40 pairs of $\mathrm{GC}$ and para-cancerous gastric mucosal tissues. (D) q-PCR analysis of MTDH mRNA levels in 40 fresh pairs of GC and para-cancerous gastric mucosal tissues. GAPDH mRNA was used as an internal control.(E) q-PCR analysis of ATG5 mRNA levels in 40 pairs of GC and para-cancerous gastric mucosal tissues. GAPDH mRNA was used as an internal control. ${ }^{* * *} \mathrm{P}<0.001$. 
MTDH enhances 5-FU resistance

MTDH protein expression levels in the normal gastric mucosa cell line (GES-1) and the 5 GC cell lines (MKN45, BGC823, HGC803, AGS, and SGC7901) were examined by western blotting (Fig. 2A). The AEG-1 mRNA levels of the abovementioned cell lines were examined by q-PCR (Fig. 2B). We found that both AEG-1 mRNA and MTDH protein expression levels were low in the normal gastric mucosa GES- 1 cells and moderate in the MKN45 cells.Therefore, we choose the MKN45 cells to conduct the following MTDH overexpression and knockdown experiments. In our previous study, we established a stable shMTDH-MKN45 cell line [14].

The GC MKN45 cell line was used to analyse the relationship between MTDH expression and responsiveness to 5-FU chemotherapy. After $48 \mathrm{~h}$ of treatment with different doses of 5-FU $(1,10,100$, and $1000 \mu \mathrm{mol} / \mathrm{L}$ ), chemotherapeutic sensitivity was assessed with CCK-8. The experiment showed that shMTDH cells were more sensitive to 5 -FU than shNC cells, whereas the cells overexpressing MTDH were more resistant than their contrasting cells (Fig. 2C). The IC50 values of 5 -FU in the abovementioned 4 cell lines were $108 \pm 15.34,30.25 \pm 2.45,89 \pm 13.45$, and $707.3 \pm 30.33 \mu \mathrm{M}$, respectively (Fig. 2D). The drug resistance-related gene P-gp was examined by western blotting of the MKN45 cell lines with different MTDH expression levels (Fig. 2E). The efficiency of MTDH knockdown and overexpression was assessed by q-PCR analysis (Fig. 2F). The results indicated that MTDH induced 5-FU drug resistance in the GC MKN45 cell line.

\section{MTDH induces active autophagyin GC}

Next, we examined whether AEG-1 induced the accumulation of LC3-II and promoted autophagy. Compared to the LC3-II levels in the control MKN45 cells, those in the shMTDH cell line were decreased and those in the MTDH-overexpressing MKN45 cells were increased based on western blotting analysis [28] (Fig. 3A). The same results were found by q-PCR (Fig. 3B). Transmission electron microscopy was used to observe membrane-bound structures sequestering cellular components (called autophagosomes) when autophagy occurred [16]. The autophagosomes were assessed in the MKN45 knockdown and overexpressing cells and vector-transfected cells (Fig. 3C). The number of autophagosomes was increased in the MTDH-overexpressing cells and decreased in the MTDH-knockdown cells (Fig. 2D). Then, the mRFP-eGFP-LC3 plasmid was transfected into the 4 cell lines mentioned above; after 48 $\mathrm{h}$, detection of green fluorescence (eGFP-LC3) indicated the formation of autophagosomes, whereas red fluorescence (mRFP-LC3) was an indicator of autophagolysosome formation 


\section{Cellular Physiology Cell Physiol Biochem 2018;46:847-859 \begin{tabular}{l|l} 
DOI: 10.1159/000488742 & and Biochemistry 2018 The Author(s). Published by S. Karger AG, Basel \\
Published online: April 06, 2018 & $\begin{array}{l}\text { www.karger.com/cpb } \\
\text { and }\end{array}$
\end{tabular}

because eGFP fluorescence was quenched due to the acidic conditions associated with the autophagolysosomes [29]. Punctate fluorescent patterns were observed by confocal microscopy to be strengthened in the MTDH-overexpressing cells, but were weakened in the MTDH-knockdown cells (Fig. 2E). The number of punctate fluorescent spots was counted and found to be increased in the MTDH-overexpressing cells and decreased in the MTDHknockdown cells (Fig. 2F).These data revealed that MTDH induced autophagy in the GC MKN45 cell line.

MTDH enhances 5-FU resistance through autophagy

We confirmed that the expression level of MTDH was closely related to 5-FU resistance and autophagy. Many studies have shown that autophagy can induce resistance to chemotherapy; thus, we explored whether MTDH promoted 5-FU resistance through autophagy. Knockdown of the autophagy essential gene ATG5 revealed that MTDH induced 5-FU resistance by promoting autophagy. The efficiency of ATG5 knockdown was detected by western blotting and q-PCR analysis (Fig. 4A and 4B). The ATG5 gene was knocked down, and the cells were transfected with the LC3-GFP plasmid. After 48 h, LC3-GFP aggregation in the cytoplasm was reduced based on confocal microscopy analysis (Fig. 4C). The drug resistance-related gene P-gp was reduced in both the western blotting and q-PCR assays (Fig. 4D). We found that MTDH induced 5-FU resistance (Fig. 2C) when the ATG5 gene was knocked down. Using CCK-8 detection, the sensitivity of

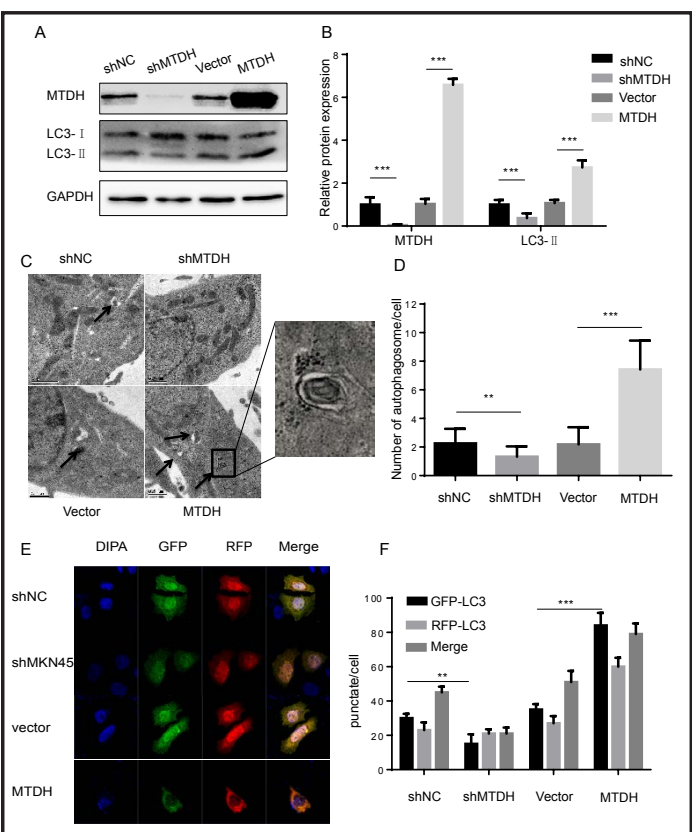

Fig. 3. MTDH activates autophagy in GC. (A and B) Following MTDH knockdown or overexpression in MKN45 cells, LC3 expression was analysed by western blotting. GAPDH protein expression was used as an internal control. (C)MTDH knockdown and overexpression in MKN45 and control cells were assessed by electron microscopy. Scale bar, $2 \mu \mathrm{m}$. (D) The number of autophagosomes was determined in MKN45 control cells after MTDH knockdown and overexpression compared to the control cells. The values are the means \pm SDs of 3 independent experiments. (E) The abovementioned cells were transfected with the mRFP-eGFP-LC3 plasmid for $48 \mathrm{~h}$, and the localization of LC3 was examined by confocal microscopy (400x). (F) Autophagosome formation was quantified through colour-coded punctate fluorescence analysis. Columns, means of punctate fluorescence in 100 cells; bars, SDs. ${ }^{* *} \mathrm{P}<0.01,{ }^{* * *} \mathrm{P}<0.001$. MTDH-overexpressing cells to 5-FU was found to be weakened, whereas sensitivity to 5-FU was weakened in cells in which ATG5 was not knocked down (Fig. 4E). Collectively, these results showed that MTDH induced autophagy and then promoted resistance to 5-FU chemotherapy in the GC MKN45 cell line.

\section{MTDH enhances AMPK-ATG5 activation}

When MTDH was knocked down in the MKN45 cell line, AMPK phosphorylation at Thr172 was reduced, and MTDH overexpression was enhanced, according to western blotting analysis (Fig. 5A). To explore whether AMPK played an essential role in autophagy through MTDH, we designed an AMPK knockdown sequence (5'-GCGUGUACGAAGGAAGAAUTT- ${ }^{\prime}$ ) that was transfected into the MKN45 cell line; then, the proteins were extracted for analysis. After $48 \mathrm{~h}$, the presence of LC3-II was decreased in western blotting analysis. We transfected an AMPK-siRNA into the MTDH and vector MKN45 cells and found that the expression level of ATG5 decreased concomitant with LC3-II transformation (Fig. 5C). The results obtained 


\section{Cellular Physiology Cell Physiol Biochem 2018;46:847-859

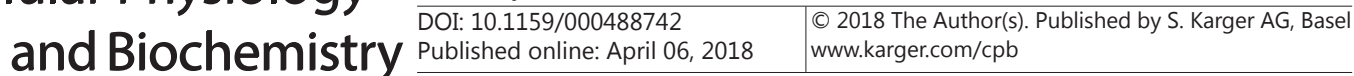

Pei et al.: Autophagy Facilitates Chemotherapy Resistance in GC

Fig. 4. MTDH enhances 5 -FU resistance through autophagy. (A and B) MKN45 cells were transfected with ATG5-targeting and negative control siRNAs. At $48 \mathrm{~h}$ later, ATG5 protein and mRNA expression levels were determined by western blotting and q-PCR, respectively. GAPDH protein and mRNA expression levels were used as the respective internal controls. Data are the means \pm SDs. (C) MKN45 cells overexpressing MTDH or control MKN45 cells were transfected with the GFPLC3 plasmid for $48 \mathrm{~h}$, and then LC3 localization was examined by confocal microscopy (400x). Autophagosome formation was quantified through green punctate fluorescence. Columns, mean of punctate fluorescence in 100 cells; bars, SDs. (D) The abovementioned cells were transfected with an ATG5-targeting siRNA. After $48 \mathrm{~h}$, differential P-gp expression was confirmed by western blotting. GAPDH mRNA and protein expression levels were used as the internal controls. (E) The effects of 5-FU on cell viability were examined following ATG5 knockdown in MKN45 cells with

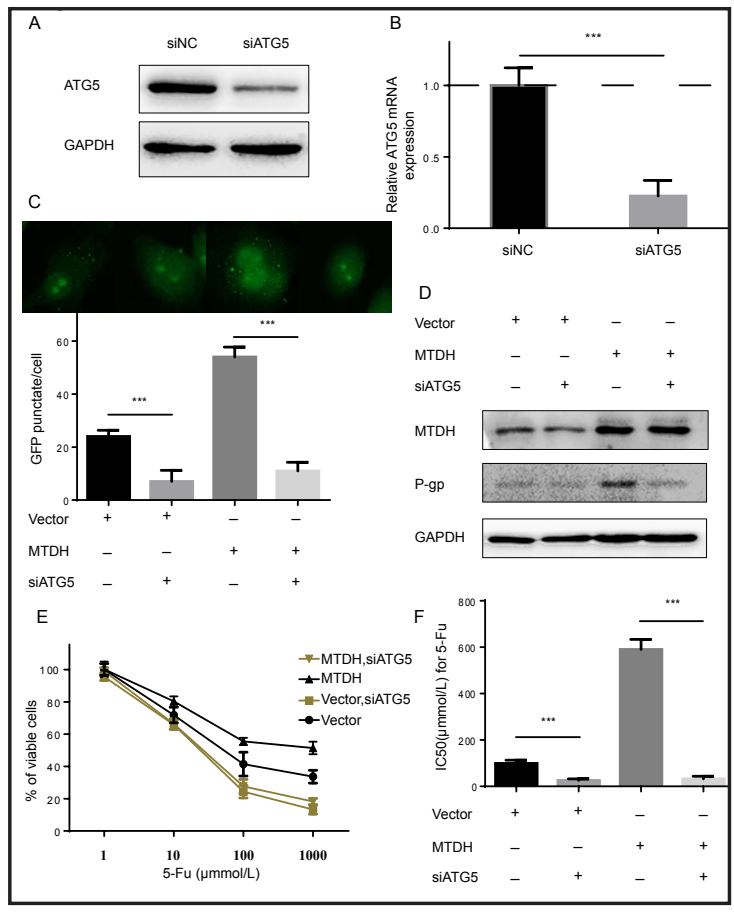
or without MTDH overexpression. The cells were treated with increasing concentrations of 5-FU (1, 10, 100, and $1000 \mu \mathrm{mol} / \mathrm{L})$. At $48 \mathrm{~h}$ later, the CCK-8 assay was used to quantify the viable cells. Point, mean of 3 experiments; bars, SDs. (F) The IC50 values were determined via the CCK-8 assay after 5-FU treatment; Columns, means of triplicate samples; bars, SDs. ${ }^{* *} \mathrm{P}<0.01,{ }^{* * *} \mathrm{P}<0.001$.

when the cells were treated with compound C $(10 \mu \mathrm{mol} / \mathrm{L}$ for $2 \mathrm{~h})$, which is an AMPK inhibitor, were similar to those obtained with the cells transfected with AMPK-siRNA [30, 31], with decreases in ATG5 expression and LC3-II transformation (Fig. 5D). These data indicated that MTDH promoted the phosphorylation of AMPK at Thr-172, activated the corresponding pathway, inhibited ATG5 expression, and finally induced autophagy. Therefore, AMPK played a significant role in the MTDH-induced autophagy process.

\section{PDX tumour models reveal that MTDH promotes 5-FU resistance}

To investigate whether MTDH was associated with GC 5-FU resistance, we manufactured xenograft models using gastric tumours, including 5 cases with high MTDH expression and 5 cases with low MTDH expression; each case was performed in 6 replicates. Three mice for each case were administered 5-FU by intraperitoneal injection $(5 \mathrm{mg} / \mathrm{kg})$ once every 2 days, and the other 3 mice were injected with $0.9 \%$ normal saline (NS) intraperitoneally. Thus, we had the following 4 groups of mice: high MTDH expression and 5-FU injection; high MTDH expression and NS injection; low MTDH expression and 5-FU injection; and low MTDH expression and NS injection.

Tumour growth in the PDX-bearing mice treated with 5-FU was inhibited in contrast to the NS injection group. The PDX tumours generated from the high MTDH-expressing cases were more strongly inhibited than the tumours from the low MTDH-expressing cases (Fig. 6C and 6D).

We performed immunohistochemistry staining analysis of the PDX tumour models and found that MTDH was expressed at different levels in the different tumours as follows: 5 PDX tumours with low MTDH levels $(\mathrm{H}$-score $<100)$ and 5 PDX tumours with high MTDH levels (H-score > 200) (Fig. 6E and 6F).

The PDX tumours generated from the high MTDH-expressing cases showed stronger 5-Fu resistance. 
Fig. 5. MTDH enhances AMPK/ATG5 pathway activation. (A and B) MTDH knockdown or overexpression, AMPK phosphorylation, and ATG5 expression were analysed by western blotting. GAPDH protein expression was used as an internal control. (C and D) MTDHoverexpressing or control MKN45 cells were transfected with an ATG5-targeting siRNA. After 48 h, AMPK and AMPK phosphorylation levels were confirmed by western blotting. GAPDH protein expression was used as an internal control. (E and F) MTDHoverexpressing or control MKN45 cells were transfected with an AMPK-targeting siRNA or control siRNA. After 48 h, AMPK expression, AMPK phosphorylation, ATG5 expression, and LC3-II expression were confirmed by western blotting. GAPDH protein expression was used as an internal control. (G and H) MTDHoverexpressing or control MKN45 cells were treated with $10 \mu \mathrm{M}$ compound $\mathrm{C}$ for $2 \mathrm{~h}$. After 48 h, AMPK, AMPK phosphorylation, ATG5, and LC3-II levels were confirmed by western blotting. GAPDH protein expression was used as an internal control. ${ }^{* *} \mathrm{P}<0.01,{ }^{* * *} \mathrm{P}<0.001$.

Fig. 6. PDX tumour models reveal that MTDH promotes 5-FU resistance. (A and B) Tumour volumes of mice with low and high MTDH expression following injection with 5-FU or NS. (C) Tumour proliferation in the MTDH high-expression nude mouse model injected with 5-FU or NS. (D) Tumour proliferation in the MTDH low-expression nude mouse model injected with 5-FU or NS. (E) Immunohistochemistry staining of the PDX tumours generated from the 5 high and 5 low MTDH expression cases; scale bar, 100 $\mu \mathrm{m}(200 \times)$. (D) MTDH H-scores of the PDX tumours generated from the 5 high and 5 low MTDH expression GC cases. ${ }^{* * *} \mathrm{P}<0.001$.
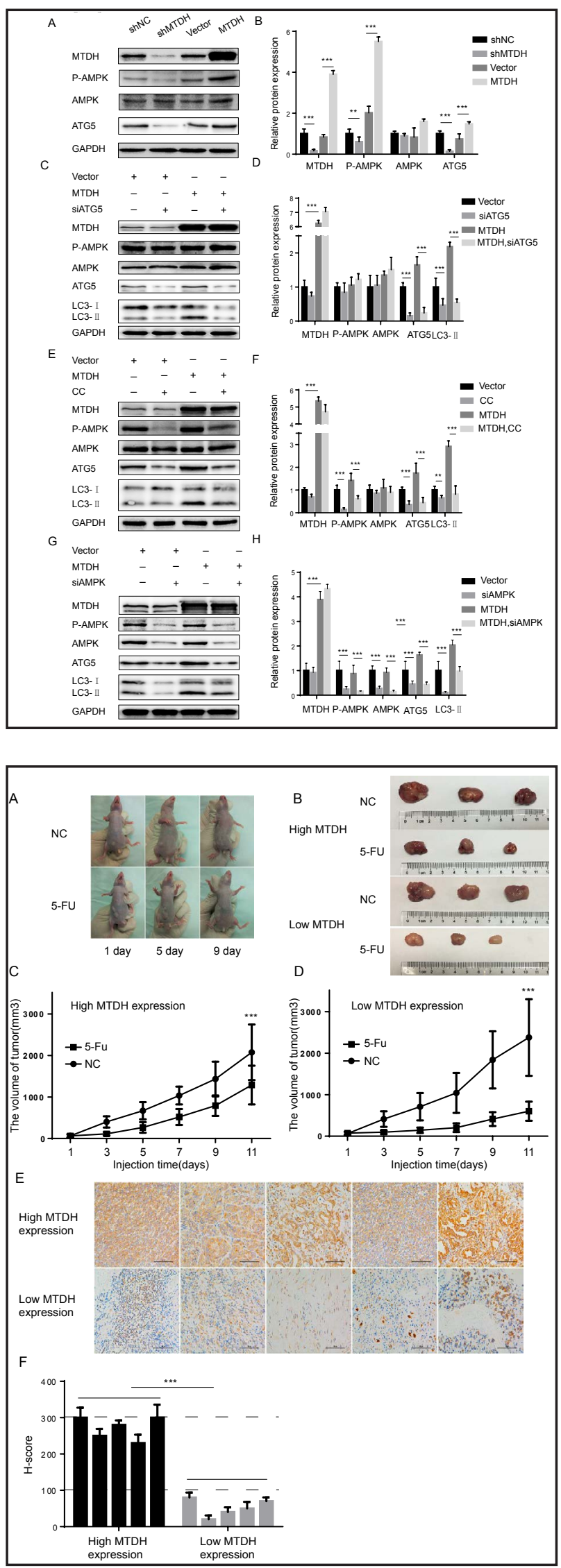


\section{Cellular Physiology Cell Physiol Biochem 2018;46:847-859 \\ \begin{tabular}{c|c} 
DOI: 10.1159/000488742 & O 2018 The Author(s). Published by S. Karger AG, Basel \\
www.karger.com/cpb
\end{tabular}}

Pei et al.: Autophagy Facilitates Chemotherapy Resistance in GC

\section{Discussion}

One vital cause of tumour formation is the activation of an oncogene, such as MTDH. Our previous study showed that MTDH played a significant role in invasion and metastasis during GC progression. MTDH has been reported to play central roles in autophagy, proliferation, and drug resistance $[32,33]$.

Through the accumulation of clinical GC specimens, we found that MTDH was highly expressed in GC tissues compared with adjacent normal gastric mucosal tissues. One of the major barriers to cancer treatment is drug resistance. Overexpression of the membrane pump P-gp is one of the main mechanisms underlying chemoresistance in cancer cells and can increase the intracellular calcium concentration. In our study, MTDH overexpression resulted in the high expression of the multidrug resistance gene P-gp. Thus, our study partially elucidated the mechanism by which MTDH facilitated GC drug resistance. The indication of novel targets for the chemoresistance process of GC is promising.

Multiple studies have revealed that MTDH plays a crucial role in 5-FU, doxorubicin, cisplatin, etoposide, and paclitaxel chemoresistance [34], and the underlying mechanisms were found. Yoo et al. reported that MTDH conferred 5-FU resistance by increasing the expression of the initial and rate-limiting enzyme of 5-FU catabolism (dihydropyrimidine dehydrogenase) [35]. In their subsequent study, the authors asserted that MTDH/AEG-1 activated the expression of MDR1 protein [22].Qian et al [36]. and Meng et al [37]. found that MTDH mediated drug resistance by inhibiting apoptosis. Moreover, MTDH regulated microRNAs to induce chemoresistance [38]. To determine whether MTDH promoted 5-FU resistance, we knocked down and overexpressed MTDH and thenconducted CCK-8 and western blotting analyses. We found that MTDH promoted drug resistance in the GC MKN45 cell line by regulating the expression of the MDR1 protein P-gp. To explore further how MTDH enhanced 5-FU resistance, we established PDX mouse models and showed that 5-FU injection slowed tumour growth in mice with tumours with low MTDH expression. Thus, MTDH enhanced 5-FU resistance.

Autophagy is a process by which a cytoplasmic protein or an organelle that has consumed its own cytoplasm is encapsulated into a vesicle fused with lysosomes to form autophagolysosomes and finally degrade the contents [39]. In our study, we found that autophagy-related LC3-II was increased concomitantly with the increased expression of MTDH detected by western blotting. Electron microscopy and confocal microscopy showed that autophagy was positively correlated with the expression level of MTDH. Therefore, we concluded that autophagy was enhanced when MTDH was overexpressed, but weakened when MTDH was knocked down in GC.

Tumour cell death due to therapy has been shown to be suppressed through autophagy [40]. Here, we demonstrated that protective autophagy induced by MTDH was related to chemoresistance. Our study proved that the overexpression of MTDH resulted in an increase of protective autophagy and induced the chemosensitization of GC MKN45 cells, whereas knocking down MTDH decreased autophagy and induced resistance to chemotherapy.

Recent studies have shown that autophagy is associated with cancer drug resistance $[23,41]$. Knocking down the ATG5 gene suppresses autophagy. We found that the expression of the MDR1 protein P-gp was low at the same time. Therefore, we speculated that MTDH enhanced 5-FU resistance through autophagy. Our study is the first to demonstrate that MTDH promotes the resistance of GC MKN45 cells to 5-FU through autophagy.

Sujit et al. noted that MTDH promoted AMPK phosphorylation, increased ATG5 expression, and enhanced autophagy in normal cells [16]. However, the molecular mechanisms by which MTDH promotes GC autophagy and drug resistance have not been investigated fully. We investigated whether MTDH enhanced autophagy via the AMPK/ATG5 pathway in GC MKN45 cells. When AMPK expression was knocked down by siRNA or inhibited using compound C [42], western blotting analysis showed that ATG5 and LC3-II expression was decreased. When ATG5 was knocked down, the LC3-II level was decreased, whereas the 
AMPK and phosphorylated AMPK expression levels did not show obvious changes. Finally, we demonstrated that MTDH induced autophagy in GC MKN45 cells.

Our study had some shortcomings. We confirmed that MTDH enhanced 5-FU resistance; however, determining whether MTDH also enhances resistance to other drugs requires further exploration. Regardless, MTDH may be a target for the efficient treatment of GC drug resistance.

In summary, the study reveals distinctive aspects of MTDH function and identifies this gene as a unique regulator of autophagy, which further actives the AMPK/ATG5 pathway and induces drug resistance in GC. Thus, MTDH is expected to be a new target for GC treatment.

\section{Acknowledgements}

This work was supported by a grant from the Shanghai Municipal Health Bureau Foundation of China (201540202).

\section{Disclosure Statement}

No conflict of interests exists.

\section{References}

1 Park JY, Von KL, Herrero R: Prevention strategies for gastric cancer: a global perspective. Clinical Endoscopy 2014;47:478-489.

2 Ji R, Zhang B, Zhang X, Xue J, Yuan X, Yan Y, Wang M, Zhu W, Qian H, Xu W: Exosomes derived from human mesenchymal stem cells confer drug resistance in gastric cancer. Cell Cycle 2015;14:2473-2483.

-3 Brown D M, Ruoslahti E: Metadherin, a cell surface protein in breast tumors that mediates lung metastasis. Cancer Cell 2004;5:365-374.

4 Sutherland HG, Lam YW, Briers S, Lamond AI, Bickmore WA: 3D3/lyric: a noveltransmembrane protein of the endoplasmic reticulum and nuclear envelope, which is also present in the nucleolus. Exp Cell Res 2004;294:94-105.

- 5 Hu G, Wei Y, Kang Y: The multifaceted role of MTDH/AEG-1 in cancer progression. Clin Cancer Res 2009;15:5615-5620.

6 Sarkar D, Fisher PB: AEG-1/MTDH/LYRIC: clinical significance. Adv Cancer Res 2013;120:39-74.

7 He R, Gao L, Ma J, Peng Z, Zhou S, Yang L, Feng Z, Dang Y, Chen G: The essential role of MTDH in the progression of HCC: a study with immunohistochemistry, TCGA, meta-analysis and in vitro investigation. Am J Transl Res 2017;9:1561-1579.

8 Luo Y, Zhang X, Tan Z, Wu P, Xiang X, Dang Y, Chen G: Astrocyte Elevated Gene-1 as a Novel Clinicopathological and Prognostic Biomarker for Gastrointestinal Cancers: A Meta-Analysis with 2999 Patients. Plos One 2015;10:e0145659.DOI:10.1371/journal.pone.0145659.

-9 Huang Y, Li LP: Progress of cancer research on astrocyte elevated gene-1/Metadherin (Review). Oncol Lett 2014;8:493-501.

10 Yu J, Wang JG, Zhang L, Yang HP, Wang L, Ding D, Chen Q, Yang WL, Ren KH, Zhou DM: MicroRNA-320a inhibits breast cancer metastasis by targeting metadherin. Oncotarget 2016;7:38612-38625.

11 Yu Z, Li ZY, Hou XX, Xiao W, Luo YH, Ying YP, Gang C: Clinical significance and effect of AEG-1 on the proliferation, invasion, and migration of NSCLC: a study based on immunohistochemistry, TCGA, bioinformatics, in vitro and in vivo verification. Oncotarget 2017;8:16531-16552.

12 Liu HY, Liu CX, Han B, Zhang XY, Sun RP: AEG-1 is associated with clinical outcome in neuroblastoma patients. Cancer Biomark 2012;11:115-121.

13 Qian B, Yao Y, Liu C, Zhang J, Chen H, Li H: SU6668 modulates prostate cancer progression by downregulating MTDH/AKT signaling pathway. Int J Oncol 2017;50:1601-1611. 


\section{Cellular Physiology Cell Physiol Biochem 2018;46:847-859

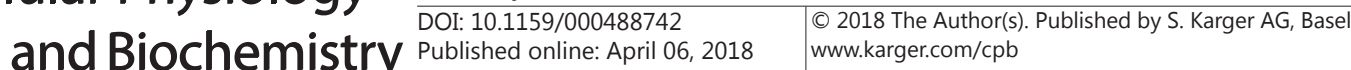

14 Du Y, Jiang B, Song S, Pei G, Ni X, Wu J, Wang S, Wang Z, Yu J: Metadherin regulates actin cytoskeletal remodeling and enhances human gastric cancer metastasis via epithelial-mesenchymal transition. Int J Oncol 2017;51:63-74.

15 Yang J, Fan B, Zhao Y, Fang J: MicroRNA-202 inhibits cell proliferation, migration and invasion of glioma by directly targeting metadherin. Oncol Rep 2017;38:1670-1678.

16 Bhutia SK, Kegelman TP, Das SK, Azab B, Su ZZ, Lee SG, Sarkar D, Fisher PB: Astrocyte elevated gene-1 induces protective autophagy. Proc Natl Acad Sci U S A 2010;107:22243-22248.

17 Shi X, Wang X: The role of MTDH/AEG-1 in the progression of cancer. Int J Clin Exp Med 2015;8:4795-4807.

18 Nestal de Moraes G, Souza PS, Costas FC, Vasconcelos FC, Reis FR, Maia RC: The Interface between BCRABL-Dependent and -Independent Resistance Signaling Pathways in Chronic Myeloid Leukemia. Leuk Res Treatment 2012;2012:671702.DOI: 10.1155/2012/671702.

19 Liu PF, Hsu CJ, Tsai WL, Cheng JS, Chen JJ, Huang IF, Tseng HH, Chang HW, Shu CW: Ablation of ATG4B Suppressed Autophagy and Activated AMPK for Cell Cycle Arrest in Cancer Cells. Cell Physiol Biochem 2017;44:728-740.

20 Pyo JO, Yoo SM, Ahn HH, Nah J, Hong SH, Kam TI, Jung S, Jung YK: Overexpression of Atg5 in mice activates autophagy and extends lifespan. Nat Commun 2013;4:2300.DOI: 10.1038/ncomms3300.

21 Wan L, Hu G, Wei Y, Yuan M, Bronson RT, Yang Q Siddiqui J, Pienta KJ, Kang Y: Genetic ablation of metadherin inhibits autochthonous prostate cancer progression and metastasis. Cancer Res 2014;74:53365347.

22 Yoo BK, Chen D, Su ZZ, Gredler R, Yoo J, Shah K, Fisher PB, Sarkar D: Molecular mechanism of chemoresistance by astrocyte elevated gene-1. Cancer Res 2010;70:3249-3258.

23 Zeng X, Zhao H, Li Y, Fan J, Sun Y, Wang S, Wang Z, Song P, Ju D: Targeting Hedgehog signaling pathway and autophagy overcomes drug resistance of BCR-ABL-positive chronic myeloid leukemia. Autophagy 2015;11:355-372.

24 Du C, Yi X, Liu W, Han T, Liu Z, Ding Z, Zheng Z, Ying P, Yuan J, Han Y: MTDH mediates trastuzumab resistance in HER2 positive breast cancer by decreasing PTEN expression through an NFKB-dependent pathway. BMC Cancer. 2014;14:869-881.

25 Burman C, Ktistakis NT: Autophagosome formation in mammalian cells. Semin Immunopathol 2010;32:397-413.

26 Noch EK, Khalili K: The role of AEG-1/MTDH/LYRIC in the pathogenesis of central nervous system disease. Adv Cancer Res 2013;120:159-192.

27 Li WF, Ou Q, Dai H, Liu CA: Lentiviral-Mediated Short Hairpin RNA Knockdown of MTDH Inhibits Cell Growth and Induces Apoptosis by Regulating the PTEN/AKT Pathway in Hepatocellular Carcinoma. Int J Mol Sci 2015;16:19419-19432.

-28 Yang M, Gu YY, Peng H, Zhao M, Wang J, Huang SK, Yuan XH, Li J, Sang JL, Luo Q: NAIF1 inhibits gastric cancer cells migration and invasion via the MAPK pathways. J Cancer Res Clin Oncol 2015;141:1-11.

29 Saribas AS, Khalili K, Sariyer IK: Dysregulation of autophagy by HIV-1 Nef in human astrocytes. Cell Cycle 2015;14:2899-2904.

30 Luo S, Garcia-Arencibia M, Zhao R, Puri C, Toh PP, Sadiq O, Rubinsztein DC: Bim inhibits autophagy by recruiting Beclin 1 to microtubules. Mol Cell 2012;47:359-370.

-31 Zhao W, Peng F, Shu M, Liu H, Hou X, Wang X, Ye J, Zhao B, Wang K, Zhong C: Isogambogenic Acid Inhibits the Growth of Glioma Through Activation of the AMPK-mTOR Pathway. Cell Physiol Biochem 2017;44:1381-1395.

32 Meng X, Thiel KW, Leslie KK: Drug resistance mediated by AEG-1/MTDH/LYRIC. Adv Cancer Res 2013;120:135-157.

33 Yang J, Fan B, Zhao Y, Fang J: MicroRNA-202 inhibits cell proliferation, migration and invasion of glioma by directly targeting metadherin. Oncol Rep 2017;38:1670-1678.

34 Hu G, Chong RA, Yang Q Wei Y, Blanco MA, Li F, Reiss M, Au LS, Haffty BG, Kang Y: MTDH Activation by 8q22 Genomic Gain Promotes Chemoresistance and Metastasis of Poor-Prognosis Breast Cancer. Cancer Cell 2009;15:9-20.

35 Yoo BK, Gredler R, Vozhilla N, Su ZZ, Chen D, Forcier T, Shah K, Saxena U, Hansen U, Fisher PB, Sarkar D: Identification of genes conferring resistance to 5-fluorouracil. Proc Natl Acad Sci U S A 2009;106:1293812943. 


\section{Cellular Physiology Cell Physiol Biochem 2018;46:847-859

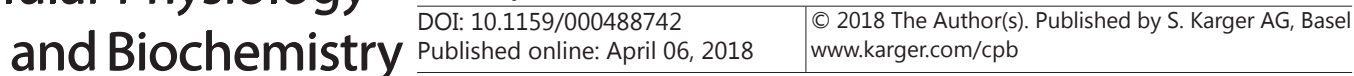

Pei et al.: Autophagy Facilitates Chemotherapy Resistance in GC

36 Qian BJ, Yan F, Li N, Liu QL, Lin YH, Liu CM, Luo YP, Guo F, Li HZ: MTDH/AEG-1-based DNA vaccine suppresses lung metastasis and enhances chemosensitivity to doxorubicin in breast cancer. Cancer Immunol Immunother 2011;60:883-893.

37 Meng X, Brachova P, Yang S, Xiong Z, Zhang Y, Thiel KW, Leslie KK: Knockdown of MTDH Sensitizes Endometrial Cancer Cells to Cell Death Induction by Death Receptor Ligand TRAIL and HDAC Inhibitor LBH589 Co-Treatment. Plos One 2011;6:e20920.DOI:10.1371/journal.pone.0020920.

-38 Huang S, Wu B, Li D, Zhou W, Deng G, Zhang K, Li Y: Knockdown of astrocyte elevated gene-1 inhibits tumor growth and modifies microRNAs expression profiles in human colorectal cancer cells. Biochem Biophys Res Commun 2014;444:338-345.

39 Abeliovich $\mathrm{H}$ : Guidelines for the use and interpretation of assays for monitoring autophagy, Haematologica2012;12:1-222.

40 Li J, Hou N, Faried A, Tsutsumi S, Takeuchi T, Kuwano H: Inhibition of Autophagy by 3-MA Enhances the Effect of 5-FU-Induced Apoptosis in Colon Cancer Cells. Ann Surg Oncol 2009;16:761-771.

41 Bhutia SK, Kegelman TP, Das SK, Azab B, Su ZZ, Lee SG, Sarkar D, Fisher PB: Astrocyte elevated gene-1 activates AMPK in response to cellular metabolic stress and promotes protective autophagy. Autophagy 2011;7:547-548.

42 Carling D, Thornton C, Woods A, Sanders MJ: AMP-activated protein kinase: new regulation, new roles? Biochem J 2012;445:11-27. 